\title{
Production, analyse et applications des huiles végétales en Afrique
}

\author{
César KAPSEU \\ Équipe de recherche Réaction Extraction Sucrerie \\ Séchage Huilerie (RESH), Département de génie \\ des procédés et d'Ingénierie, Ecole nationale \\ supérieure des sciences agro-industrielles, \\ Université de Ngaoundéré (Cameroun), \\ BP 455 Ngaoundéré \\ <kapseu@yahoo.fr>
}

\begin{abstract}
This paper analyses the evolutions of the different needs related to the food and non food uses of conventional oil crops (palm, cotton, groundnuts), immerging and marketable oil culture (shea) as well as the domestic oil cultures (Canarium, safou). Africa accounts for about $6.5 \%$ of the total World production of palm oil. In the last few years palm oil has witnessed an evolution in Africa through the diversification of its uses. A lot has also been done on the use of palm oil as bio-fuels. The production of cotton seed oil has equally witnessed changes from press extraction followed by solvent extraction to direct extraction with pure solvents followed by neutralisation in an appropriate medium as major innovations. West Africa produces about $50 \%$ of the total groundnuts production in Africa. Small scale processing of groundnuts is more popular than industrial processing. This is justified by the diverse uses of the different groundnut byproducts. The most remarkable innovations concern the emerging oil cultures such as shea butter. In fact the incorporation of $5 \%$ shea butter in chocolate formulations has given an added value to shea. Techniques have been put in place for improving on the production methods and quality of the butter. The evolution in this sector is better illustrated by the putting in place of an indirect solar dryer and a vertical manual screw press. The big handicap that slows down evolution remains at the level of the transfer of technology to the rural milieu. Problems on the transfer preservation of Canarium were resolved by preserving them in appropriate media and conditions. Dried safou fruits can now be found in the market. This illustrates the appropriation of technology by small and medium sized enterprises.
\end{abstract}

Key words: palm, cotton, peanut, shea, safou, Canarium, fatty acids, triglycerides

de $49,0 \%$ pour une offre nette de 83000 tonnes. Les huiles végétales sont essentiellement fournies par les noix de palme, les graines de coton et les germes de maïs. Le pays regorge pourtant de nombreux autres oléagineux rentrant généralement dans le régime alimentaire des populations, mais inexploités à l'échelle industrielle. Ce sont entre autres : I'avocat (Persea americana), le safou (Dacryodes edulis), la noix de coco (Cocos nucifera), le sésame (Sesamum indicum), le « djansang " (Ricinodendron heudelotii), la courge (Cucumeropsis manii), le «mbeu» (Canarium schweifurthii), le karité (Butyrospermum parkii) [3].

Les besoins du marché des oléagineux et la détection de fonctionnalités nouvelles ont suscité, au niveau national, un intérêt de la recherche scientifique et technique en direction de sources oléagineuses végétales locales, encore sous-exploitées. Les axes de recherche développés dans ce cadre concernent notamment la maîtrise des procédés d'extraction, la caractérisation des huiles extraites et la définition de leurs qualités et de leur fonctionnalité. Actuellement, outre le karité, l'aiélé ou «mbeu » et le « djansang » bénéficient de cet intérêt [4-6]. Ce travail a pour but de connaître la production, la transformation, I'utilisation ainsi que les compositions en acides gras et en triglycérides des oléoprotéagineux d'Afrique subsaharienne.

\section{Grandes cultures oléoprotéagineuses}

\section{Palme (Elaeis guineensis)}

Le palmier à huile (Elaeis guineensis) est une palmacée monocotylédone qui pousse surtout sur les sols sableux le long des côtes. Bien que des variétés naines et semi-naines existent pour cette espèce, leur taille peut atteindre 15 à $30 \mathrm{~m}$ de hauteur. Le palmier est cultivé à l'échelle industrielle. Sur le plan structural, leurs fruits présentent des différences. L'huile de palme provient de la pulpe des noix alors que l'huile de palmiste provient de l'amande séchée (palmiste). Elles entrent non seulement dans l'alimentation des populations, mais elles constituent également une source de matière première pour l'industrie de la savonnerie et de la cosmétique [7].

Le tableau 1 présente la production en tonnes de I'huile de palme pour les pays africains. Il apparaît que le Nigeria est le premier producteur suivi de la Côte-d'Ivoire et du Cameroun. Les sept premiers producteurs africains représentent en moyenne $6,5 \%$ de la production mondiale et sont parmi les vingt premiers producteurs de l'huile de palme dans le monde.

\section{Transformation}

L'huile de palme est extraite localement suivant plusieurs techniques, manuellement, artisana- 
Tableau 1. Sept premiers producteurs d'huile de palme en Afrique.

\begin{tabular}{|lrrrrr|}
\hline Pays & \multicolumn{1}{l}{1998} & \multicolumn{1}{l}{ 2002 } & \multicolumn{1}{l}{ 2005 } & \multicolumn{1}{l}{ 2006 } & \multicolumn{1}{l}{ 2007 } \\
\hline Monde & 18215637 & 25844879 & 34298016 & 37680400 & 39279920 \\
\hline Nigeria & 845000 & 961000 & 1170000 & 1287000 & 1300000 \\
\hline Côte d'Ivoire & 264403 & 252289 & 289932 & 320000 & 320000 \\
\hline Congo (RDC) & 168830 & 170000 & 175000 & 175000 & 104000 \\
\hline Cameroun & 139000 & 153121 & 154000 & 160000 & 172000 \\
\hline Ghana & 111370 & 108000 & 117000 & 121000 & 109000 \\
\hline Angola & 51000 & 50000 & 53000 & 54000 & 54500 \\
\hline Guinée & 50000 & 50000 & 50000 & 53000 & 53000 \\
\hline
\end{tabular}

Source : FOASTAT prodstat (http://faostat.fao.org/site/339/default.aspx) consulté le 20 octobre 2008. lement et industriellement à partir des noix de palme. Le procédé obéit généralement aux étapes suivantes: stérilisation (cuisson), malaxage, extraction, finition, fractionnement et raffinage. À la suite de ces étapes, les huiles de palme et de palmiste sont obtenues.

\section{Applications}

L’huile de palme est utilisée à des fins alimentaires et non alimentaires. Sur le plan alimentaire, cette huile entre dans les recettes culinaires (koki, sauce jaune, eru, kwem...). L'huile de palme occupe une place importante dans les rituels (culte). En Afrique, I'huile de palme est utilisée dans le culte des ancêtres, partagée lors des cérémonies de naissance, s'offre comme élément de dot lors des mariages et est mélangée aux potions médicamenteuses pour soulager les coliques. Au sujet des usages non alimentaires, I'huile de palme entre dans la fabrication du savon à l'échelle artisanale et industrielle. Elle sert aujourd'hui de matière première pour la fabrication des biocarburants.

L'huile de palmiste entre également dans la fabrication des médicaments et des produits cosmétiques. Sur le plan thérapeutique, I'huile de palmiste est sollicitée dans la prévention et le traitement des coliques infantiles. Elle est utilisée contre les maladies de la peau comme les érythèmes fessiers, la gale, la teigne, l'acné et les démangeaisons. Elle est aussi utilisée pour le massage du corps des nourrissons. Sur le plan cosmétique, les femmes âgées en usent habituellement pour entretenir leur peau et soulager leur rhumatisme.

\section{Coton (Gossypium sp.)}

\section{Production}

L'huile de coton fait partie des produits alimentaires les plus véhiculés dans le monde, particulièrement dans les régions tropicales. En ce qui concerne l'Afrique, elle constitue une source principale de lipides pour la population. L'huile de coton, pour être utilisée comme huile de table, doit cependant être débarrassée de certaines impuretés [8].

Le tableau 2 représente la production en tonnes de I'huile de coton pour quelques pays africains. II apparaît que le premier producteur est le Burkina-Faso suivi du Nigeria, de l'Égypte et du Mali. La production de I'huile de coton dans ces quatre pays représente $3,5 \%$ de la production mondiale.

\section{Transformation}

La transformation du coton passe par les étapes suivantes : la trituration, l'extraction et le raffinage. L'extraction se fait à la presse suivie par l'épuisement au solvant. L'huile brute est ensuite raffinée. La technologie a évolué passant de l'extraction par presse à l'extraction directe au solvant. La neutralisation se fait en milieu solvant.

\section{Applications}

Au niveau artisanal, les fibres de coton entrent dans la confection des pagnes (leppi) par les tisserands. Au niveau industriel, ces fibres sont utilisées dans la fabrication des pagnes (wax) aux motifs et couleurs chatoyants qui rappellent la tradition africaine.

L'huile de coton est utilisée dans les recettes culinaires, dans les bains de friture (beignets, croquettes) et dans la pâtisserie traditionnelle. Les sous-produits de I'huilerie de coton entrent dans la fabrication du savon (soapstock) et des aliments pour le bétail. Les tourteaux de coton revêtent une importance capitale dans les zones où les conflits entre pasteurs et éleveurs sont exacerbés par le manque de pâturage.

\section{Arachide (Arachis hypogaea)}

\section{Production}

L'arachide (Arachis hypogaea) est une légumineuse annuelle dont le fruit mûrit en terre. Ses graines servent surtout de matière première pour l'extraction des huiles utilisées comme assaisonnement en cuisine et pour produire le beurre d'arachide. Une partie appréciable de la production d'arachide est utilisée comme produit vivrier : arachides grillées consommées telles quelles ou utilisées comme condiment, notamment dans la préparation de sauces réputées. Généralement, les ventes en coque sont destinées à la consommation de bouche et les ventes en décortiqué à la confiserie. L'arachide est la sixième culture parmi les oléagineuses les plus importantes dans le monde. Elle contient 48-50 \% de corps gras, 26-28 \% de protéine et elle est riche en fibre, minéraux et vitamines. L'arachide est cultivée sur 26,4 millions d'hectares à l'échelle mondiale avec une production totale de 37,1 millions de tonnes métriques et une productivité moyenne de 1,4 tonnes à l'hectare. La part de l'Afrique dans la production mondiale d'arachide était en 1992 de $20 \%$ dont la moitié revenait à l'Afrique de l'ouest. La part de la production de l'Afrique de l'Ouest est passée actuellement à $15 \%$. Les exportations ont chuté de $55 \%$ à $20 \%$. Le Sénégal, le Soudan et le Nigeria demeurent parmi les plus grands producteurs africains d'arachide dans le monde (tableau 3). La culture de l'arachide reste une des principales sources d'emplois, de revenus et d'échanges extérieurs dans beaucoup de pays de l'Afrique de l'Ouest. Pour cela, il y a un besoin de réviser les perspectives de marché et saisir les opportunités pour aider l'Afrique de I'Ouest à reconquérir ses parts de marché $[9,10]$.

\section{Transformation}

Le procédé traditionnel d'extraction d'huile d'arachide comprend: l'égoussage, le
Tableau 2. Quatre premiers producteurs d'huile de coton en Afrique en 2006.

\begin{tabular}{|lrrrrr|}
\hline Pays & \multicolumn{1}{l}{$1 \mathbf{1 9 9 8}$} & \multicolumn{1}{l}{$\mathbf{2 0 0 2}$} & \multicolumn{1}{l}{ 2005 } & \multicolumn{1}{l}{ 2006 } & \multicolumn{1}{l|}{ 2007 } \\
\hline Monde & 52032202 & 53825804 & 69446228 & 71455708 & \multicolumn{1}{l}{72504406} \\
\hline Burkina Faso & 324557 & 439247 & 712707 & 725000 & 690000 \\
\hline Nigeria & 348000 & 428000 & 521000 & 563000 & 570000 \\
\hline Égypte & 627694 & 765612 & 560000 & 560000 & 560000 \\
\hline Mali & 518415 & 439722 & 534143 & 432466 & 414965 \\
\hline
\end{tabular}

Source : FOASTAT prodstat (http://faostat.fao.org/site/339/default.aspx) consulté le 20 octobre 2008. 
séchage, le décorticage, le toastage, le dépelliculage et dégermage, le broyage-affinage et l'extraction d'huile.

\section{Utilisations}

Les produits dérivés d'arachides sont l'huile, le tourteau, la pâte d'arachide, le beurre d'arachide et les graines elles-mêmes. L'huile d'arachide est utilisée en cuisine car elle est très stable, et résiste bien à la cuisson, même à haute température. L'huile d'arachide est composée de $17 \%$ d'acides gras saturés, $47 \%$ d'acides gras mono-insaturés et de $32 \%$ d'acides gras polyinsaturés.

Le tourteau de graines d'arachide est riche en protéines (55-60\%) et est utilisé comme complément alimentaire ou dans la préparation des aliments de sevrage. II est aussi utilisé dans la préparation des pains et d'autres aliments. Le beurre d'arachide ou beurre de cacahuètes est une pâte à tartiner faite à base de graines d'arachides.

\section{Culture émergente commerciale : le karité}

Butyrospermum parkii (G. Don) Kotschy ou Vitellaria paradoxa Gaertn., Bassia parkii G. Don est vulgairement appelé karité en français et shea en anglais et allemand. Arbre typique des savanes arborées soudaniennes, le karité est un arbre de la famille des Sapotacées, caractérisé par la présence du latex dans les organes. II peut atteindre 15 à $20 \mathrm{~m}$ de hauteur et $1 \mathrm{~m}$ de diamètre. Les fruits de karité arrivent à maturité en début

Tableau 3. Production d'arachides en milliers de tonnesprincipaux producteurs africains.

\begin{tabular}{|lll|}
\hline Pays & Tendances & Données \\
\hline Afrique du Sud & $1970-2000$ & 169,5 \\
\hline Burkina Faso & $1970-2000$ & 205 \\
\hline Cameroun & $1970-2000$ & 160 \\
\hline Congo (Rép. Dém.) & $1970-2000$ & 382 \\
\hline Égypte & $1970-2000$ & 182 \\
\hline Ghana & $1970-2000$ & 190 \\
\hline Guinée & $1970-2000$ & 182 \\
\hline Nigeria & $1970-2000$ & 2783 \\
\hline Sénégal & $1970-2000$ & 828,3 \\
\hline Soudan & $1970-2000$ & 990 \\
\hline Tchad & $1970-2000$ & 371,9 \\
\hline Zimbabwe & $1970-2000$ & 190 \\
\hline
\end{tabular}

Source : FAO (Organisation des Nations Unies pour I'alimentation et l'agriculture). d'hivernage (fin mai et jusqu'en août). Le fruit de karité est comestible, de couleur verte et de forme arrondie. II mesure en moyenne quatre à cinq centimètres de diamètre, et est constitué d'un péricarpe charnu, de quatre à huit millimètres d'épaisseur, représentant à peu près la moitié du poids du fruit. À l'intérieur de cette pulpe sucrée se trouvent une, deux et parfois trois noyaux constitués chacun d'une amande blanchâtre à deux cotylédons, protégée par une coque mince et dure de couleur beige à marron foncé (figure 1). Après séchage, $50 \%$ de la masse de l'amande sont constitués de la matière grasse qui a une température de fusion de $40^{\circ} \mathrm{C}$ et contient 5 à $7 \%$ de latex insaponifiable ou karitène [11, 12].

\section{Production}

Les données sur les rendements varient énormément. On compte 5 à $15 \mathrm{~kg}$ d'amandes non
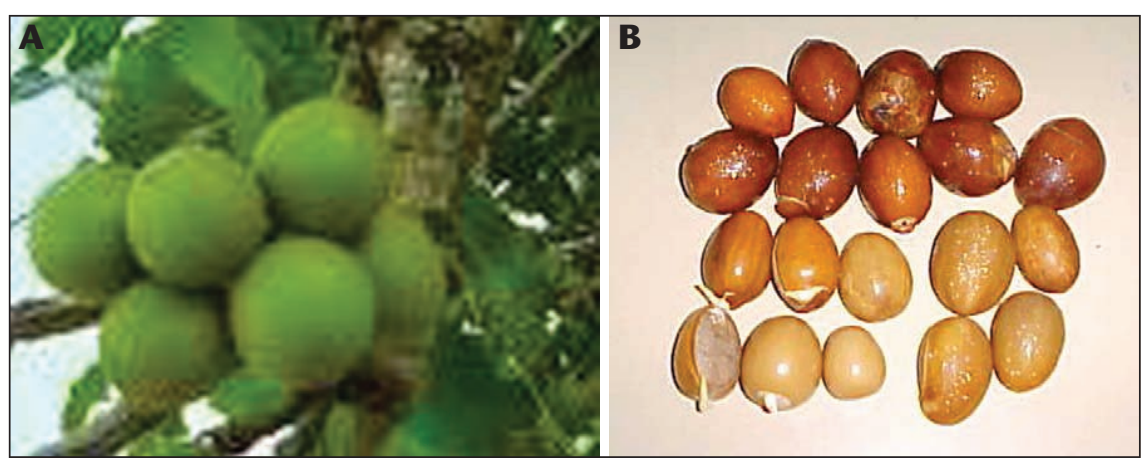

Figure 1. Les fruits (A) et graines (B) de karité.

Tableau 4. Estimations annuelles en tonnes des productions des amandes et beurre du karité en Afrique subsaharienne (Source : Lovett PNC. The shea butter value chain: production, transformation and marketing in west Africa. Tehnical Report, 2004, N² 2, 52 pp.).

\begin{tabular}{|lcccrrr|}
\hline Pays & $\begin{array}{l}\text { Potentiels } \\
\text { de production }\end{array}$ & $\begin{array}{l}\text { Quantité } \\
\text { collectée }\end{array}$ & $\begin{array}{l}\text { Quantité } \\
\text { consommée }\end{array}$ & \multicolumn{2}{l}{ Quantité exportée } \\
\cline { 1 - 2 } \cline { 6 - 7 } & & & & & Amandes & Beurre \\
\hline Mali & 250000 & 150000 & 97000 & 50000 & 3000 \\
\hline Nigeria & 250000 & 100000 & 80000 & 20000 & \\
\hline Ghana & 200000 & 130000 & 70000 & 45000 & 15000 \\
\hline Côte d'Ivoire & 150000 & 40000 & 15000 & 15000 & 10000 \\
\hline Burkina Faso & 150000 & 75000 & 35000 & 37000 & 3000 \\
\hline Soudan & 100000 & 10000 & 9800 & & 0 & 200 \\
\hline Ouganda & 70000 & 6000 & 5900 & & 0 & 100 \\
\hline Bénin & 80000 & 50000 & 14900 & 35000 & 100 \\
\hline Togo & 50000 & 40000 & 10000 & 15000 & 15000 \\
\hline Cameroun & 30000 & 5000 & 2500 & 2500 & 0 \\
\hline Guinée Conakry & 25000 & 5000 & 4500 & 450 & 50 \\
\hline
\end{tabular}

décortiquées par arbre et par an, ces chiffres dépendent beaucoup des précipitations annuelles. Mais, on connaît des arbres qui bien soignés et protégés donnent $45 \mathrm{~kg}$. Des rendements annuels par hectare de 9 à 17 tonnes de fruits frais sont possibles; $50 \mathrm{~kg}$ fournissent $20 \mathrm{~kg}$ d'amandes séchées et $4 \mathrm{~kg}$ de beurre.

La production totale de noix de karité est difficile à estimer, car une partie importante se vend en Afrique même ou se troque contre des noix de cola et du sel. On l'estimait en 1913 en Afrique occidentale entre 200000 et 300000 t et en 1975 à 500000 t, dont seuls $4 \%$ parvenaient sur le marché mondial. En 1978/79 on indique pour le Mali 100000 t récoltées dont 50000 t exportées (valeur marchande $20 \mathrm{FCFA} / \mathrm{kg}$ ). Les principaux importateurs de karité sont I'Union européenne (UE), le Japon et les pays d'Europe de l'Est. En 1992, les importations étaient de 11900 tonnes de karité pour l'UE et de 5076 tonnes pour le Japon. 
Le tableau 4 représente la production annuelle des amandes de karité en Afrique. II en ressort que le Mali est le premier producteur des amandes suivi du Nigeria et du Ghana.

\section{Transformation}

La transformation du karité demeure, dans la plupart des cas, une activité artisanale. Les conditions paysannes de transformation sont peu contrôlées et handicapées par les moyens et les techniques de transformation. Les propriétés intrinsèques des huiles dépendent non seulement de la technique d'extraction mais aussi du soin apporté aux fruits, graines et amandes, de la collecte au stockage des huiles. Le séchage direct au soleil est I'un des handicaps du procédé car il est une technique lente, soumise aux conditions climatiques avec les risques de pollution et d'oxydation élevés. Elle est favorable à l'action des lipases qui entraîne l'augmentation des acides gras libres. Les travaux réalisés ont abouti à une voie d'amélioration du séchage au soleil.

\section{Les méthodes traditionnelles d'extraction du beurre}

II n'existe pas une méthode unique d'extraction du beurre de karité. Le procédé d'extraction varie d'une région à l'autre, d'une tribu à une autre et est fonction du genre d'utilisation de l'extrait.

Une méthode utilisée au Burkina Faso est résumée comme suit: ramassage, dépulpage, séchage, décorticage, concassage, torréfaction, pilage, laminage, barattage, recueil de la matière grasse, chauffage, séparation du beurre des impuretés et conditionnement.

Une deuxième méthode généralement pratiquée par les femmes Foulbé du Nord-Cameroun se résume par les opérations suivantes : récolte ou ramassage, dépulpage, cuisson, décorticage, pilage, broyage, barattage, recueil de la matière grasse, lavage à grande eau, chauffage, séparation du beurre des impuretés et conditionnement (figure 2). Certaines traditions gardent les amandes dans l'eau pendant 3 à 7 jours avant le pilage. Cette méthode se rapproche de celle pratiquée au Mali.

\section{Techniques d'extraction améliorées}

Pour améliorer le séchage des noix et des amandes, des séchoirs solaires indirects (figure 3) ont été conçus. L'air chauffé dans le collecteur par les rayons solaires devient moins dense et monte dans la chambre de séchage. Cet air chaud entraîne l'eau contenue dans le produit et sort par le système d'évacuation. Ce mode de séchage a l'avantage que le produit à sécher est protégé contre les insectes et la pluie. En plus, les rayons solaires n'irradient pas directement le produit à sécher. Bup et al. [13] montrent que les amandes de karité séchées dans le séchoir solaire indirect donnent le beurre de bonne qualité selon les normes de karité pour les usages cosmétiques et pharmaceutiques. Ce séchoir fonctionne aussi en convection forcée. Le ventilateur est alimenté par l'énergie solaire provenant des cellules photovoltaïques (figure 4). La régula- tion de température est aussi assurée avec les cellules photovoltaïques dans la chambre du séchage.

Les améliorations portent sur l'extraction proprement dite et le pilage (broyage) qui est remplacé par le passage des amandes entières ou préconcassées dans un broyeur (moulin) à meules, animé par un moteur. Les opérations de conservation (pré-traitements) restent inchangées.

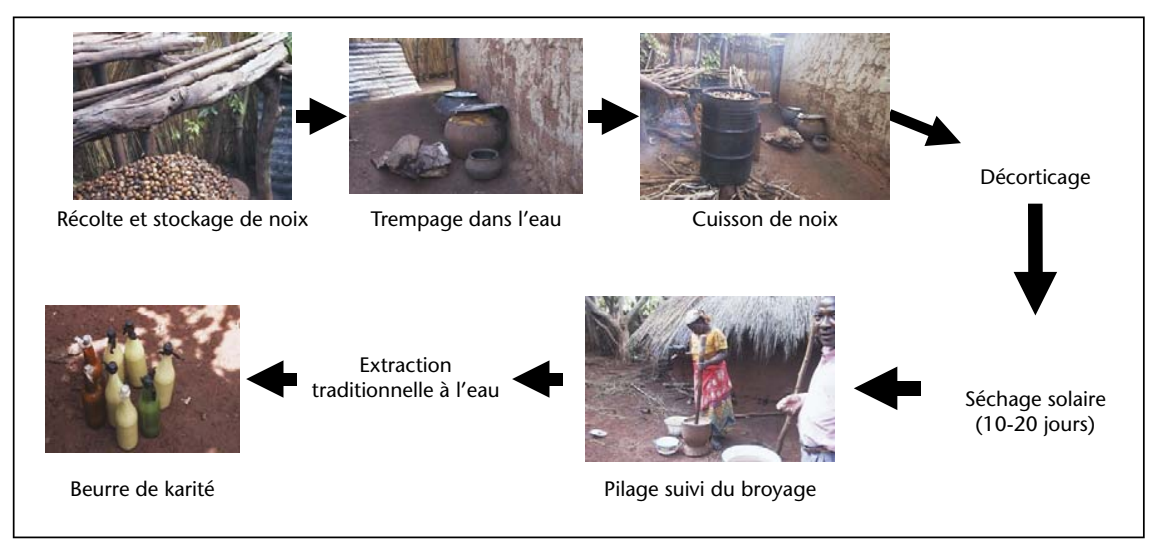

Figure 2. Procédé traditionnel de fabrication du beurre du karité au Cameroun.

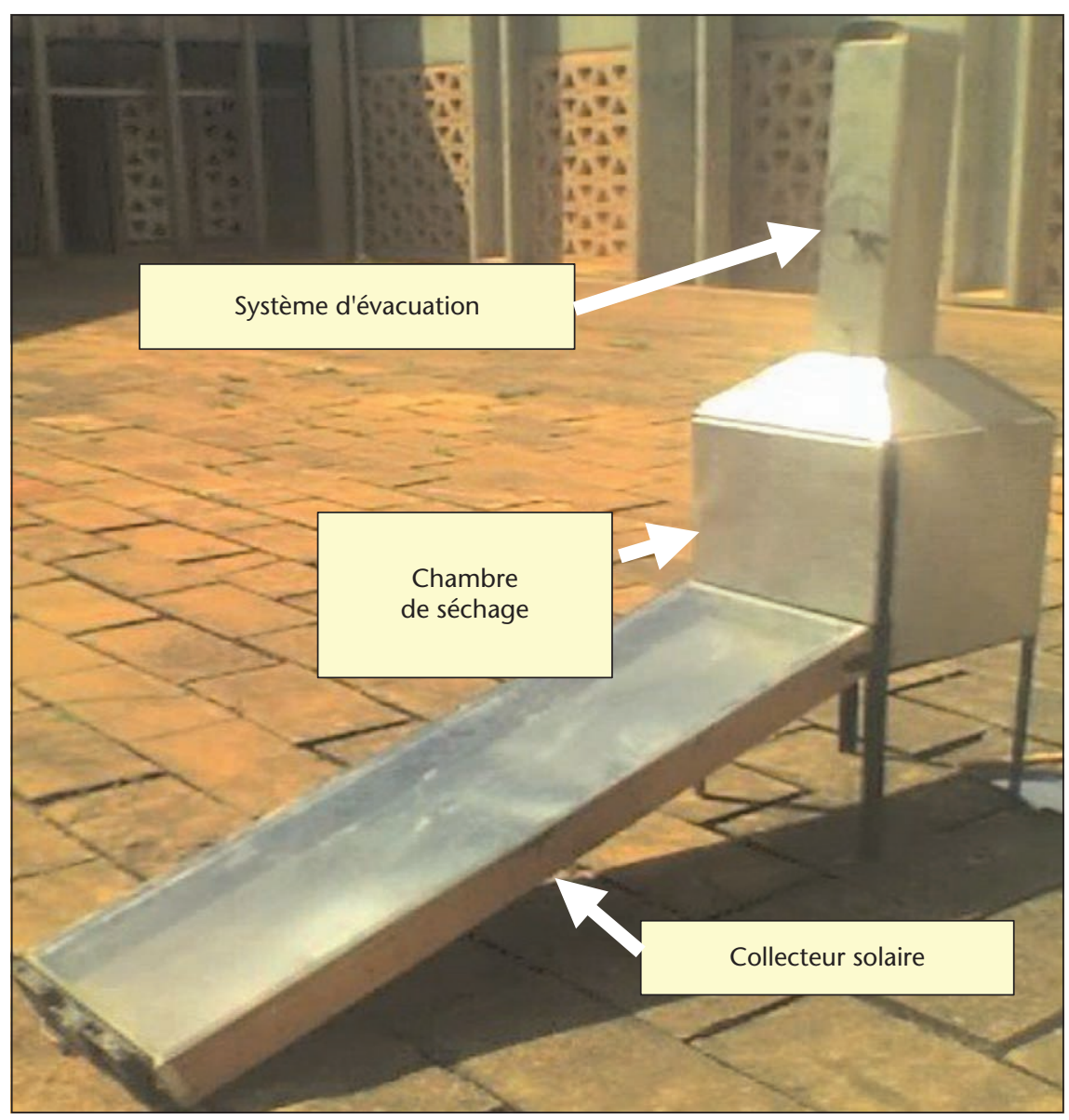

Figure 3. Séchoir solaire indirect modèle KCNDB 2006 FIS. 


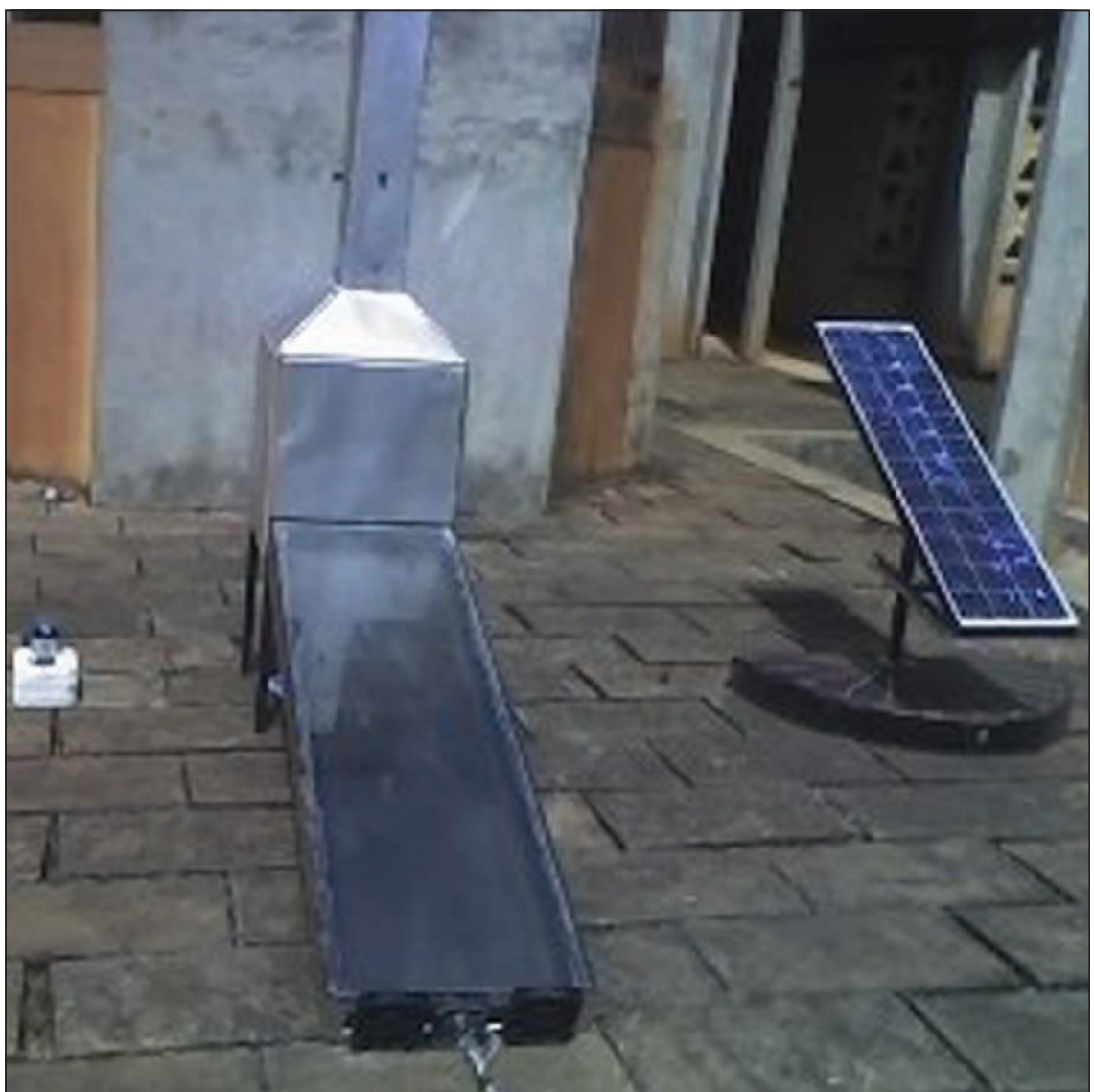

Figure 4. Séchoir solaire indirect avec cellule photovoltaïque pour la régulation de la température.

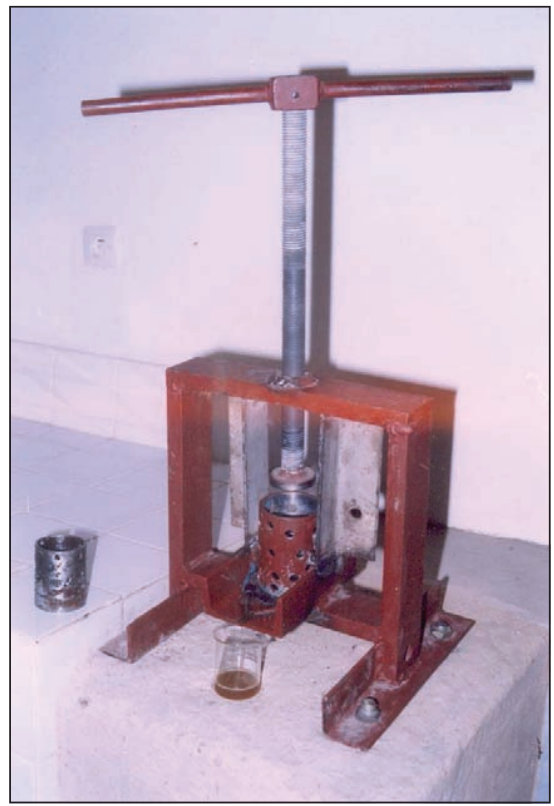

Figure 5. Presse manuelle à vis verticale pour l'extraction du beurre de karité.

L'extraction par presse. La presse à vis manuelle Segama fait la fierté des femmes au Sénégal, Gambie et au Mali; elle est adaptée au

*Absence de pic, g/100 g, matières sèches. figures 5 et 6, a été optimisé pour l'extraction du beurre de karité. L'utilisation de cette presse réduit la pénibilité du travail d'extraction. En définitive, l'intégration du séchoir solaire indirect et la presse réduit considérément le temps de fabrication du beurre de karité [14].

\section{Utilisations}

En Afrique, les produits de karité se vendent sous forme de noix séchées au soleil, d'amandes pilées en pâte (Kadanya), d'huile en estagnons, de graisse en plaques (beurre de karité) ou de margarine en boîtes.

Le produit principal du karité est son beurre qui joue un rôle économique important dans plusieurs régions. Le beurre de karité s'emploie localement dans la médecine en particulier pour les pommades ou comme cosmétique. II est utilisé comme huile de friture et crème de beauté dans les campagnes. Dans les villes, en pharmacie, en cosmétique et en pâtisserie, on I'utilise en substitution au beurre de cacao à cause de son point de fusion élevé.

\section{Les cultures domestiques}

\section{Canarium schweinfurthii}

\section{Production}

L'aiélé (Canarium schweinfurthii Engl.) est une Burcéraceae dont l'aire géographique est très répandue en Afrique. En effet, en plus du Cameroun où l'arbre pousse dans toute la zone Sud et même dans la partie Nord (Mont Atlantica), cette plante se retrouve de la Sierra Leone à I'Ouest de l'Afrique jusqu'à l'Est de I'Afrique et au Sud de I'Angola. La plante produit des fruits qui sont des oléagineux morphologiquement comparables aux olives européennes (Olea europea). Le ramollissement de la pulpe se fait à l'eau à une température de $45^{\circ} \mathrm{C}$ pendant 40 minutes. Au-delà de $70{ }^{\circ} \mathrm{C}$, les pulpes des fruits durcissent. Le durcissement peut également survenir lorsque les fruits sont stockés 5 à 7 jours après la cueillette et se caractérise par la perte de l'aptitude à ramollir. La teneur en huile de la pulpe des fruits de l'aiélé est élevée (30 à $50 \mathrm{~g} / 100 \mathrm{~g}$ ) et suscite l'intérêt des chercheurs africains (tableau 5). Pourtant,

Tableau 5. Composition chimique des huiles des pulpes des fruits de l'aiélé.

\begin{tabular}{|c|c|c|c|c|}
\hline & Crus & $\begin{array}{l}\text { Ramollis à } 45^{\circ} \mathrm{C} \\
\text { pendant } 40 \text { minutes }\end{array}$ & Durcis à l'eau & $\begin{array}{l}\text { Durcis après } \\
\text { stockage }\end{array}$ \\
\hline Teneurs en huile & $38,0 \pm 1,0$ & $44,0 \pm 1,1$ & $42,5 \pm 0,6$ & $37,5 \pm 0,9$ \\
\hline Triglycérides & 81,6 & 76,5 & 72,6 & 81,1 \\
\hline Monoglycérides & 15,3 & 16,4 & 24,6 & 14,0 \\
\hline Acides gras libres & 1,1 & $-*$ & - & 1,9 \\
\hline
\end{tabular}


rien n'est encore fait pour lutter contre le phénomène de durcissement de la pulpe des fruits (figure 7), qui entraîne des pertes énormes pour les paysans dus au fait que les fruits durcis sont tout simplement jetés. Face aux pertes postrécolte, il est important de conserver les fruits dans les saumures et de valoriser les fruits en produisant de I'huile [15-18] (figure 8).

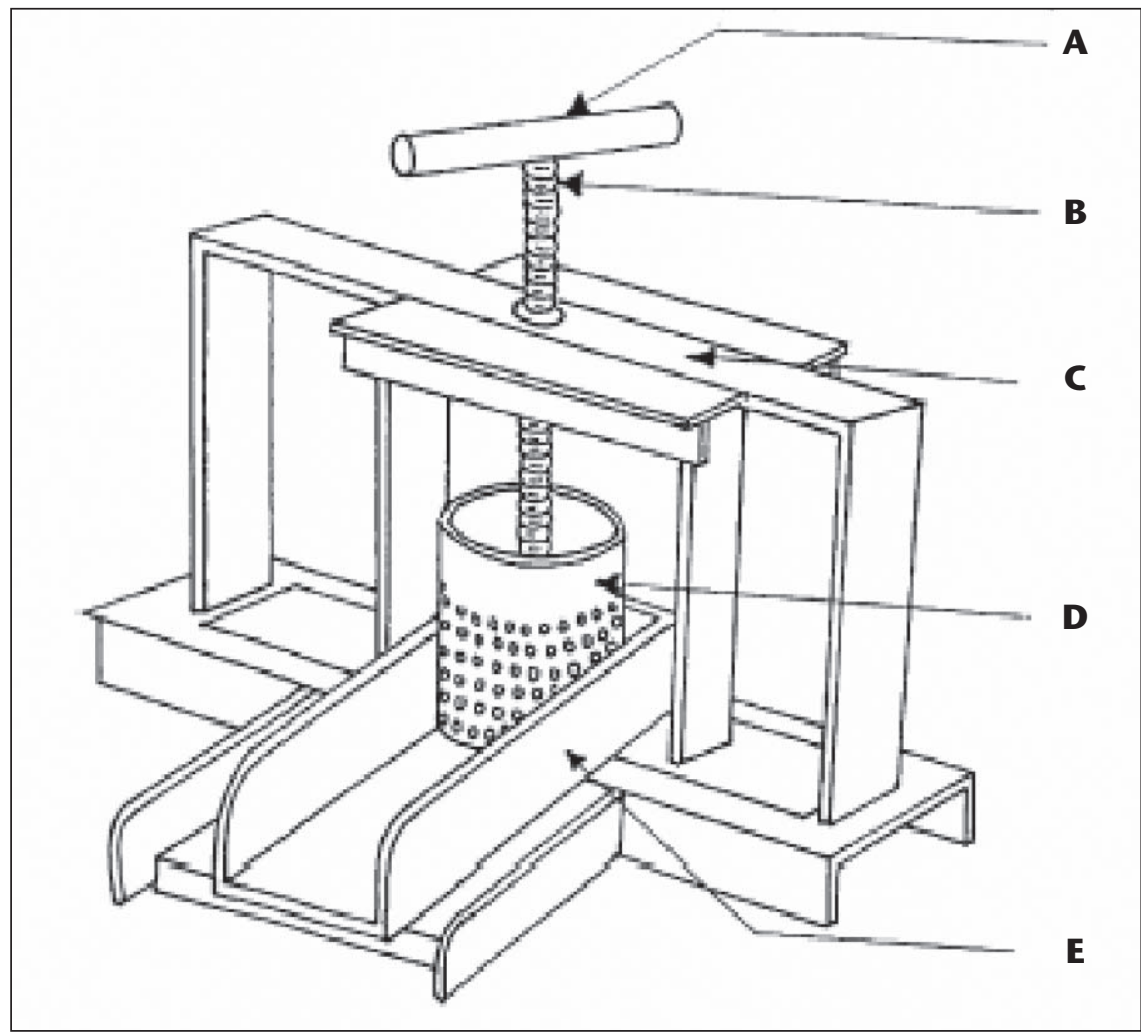

Figure 6. Presse manuelle à vis verticale (modèle CKT 2000AUF) pour l'extraction beurre de karité. A) barre, B) vis, C) cadre, D) panier-tamis, E) recette.

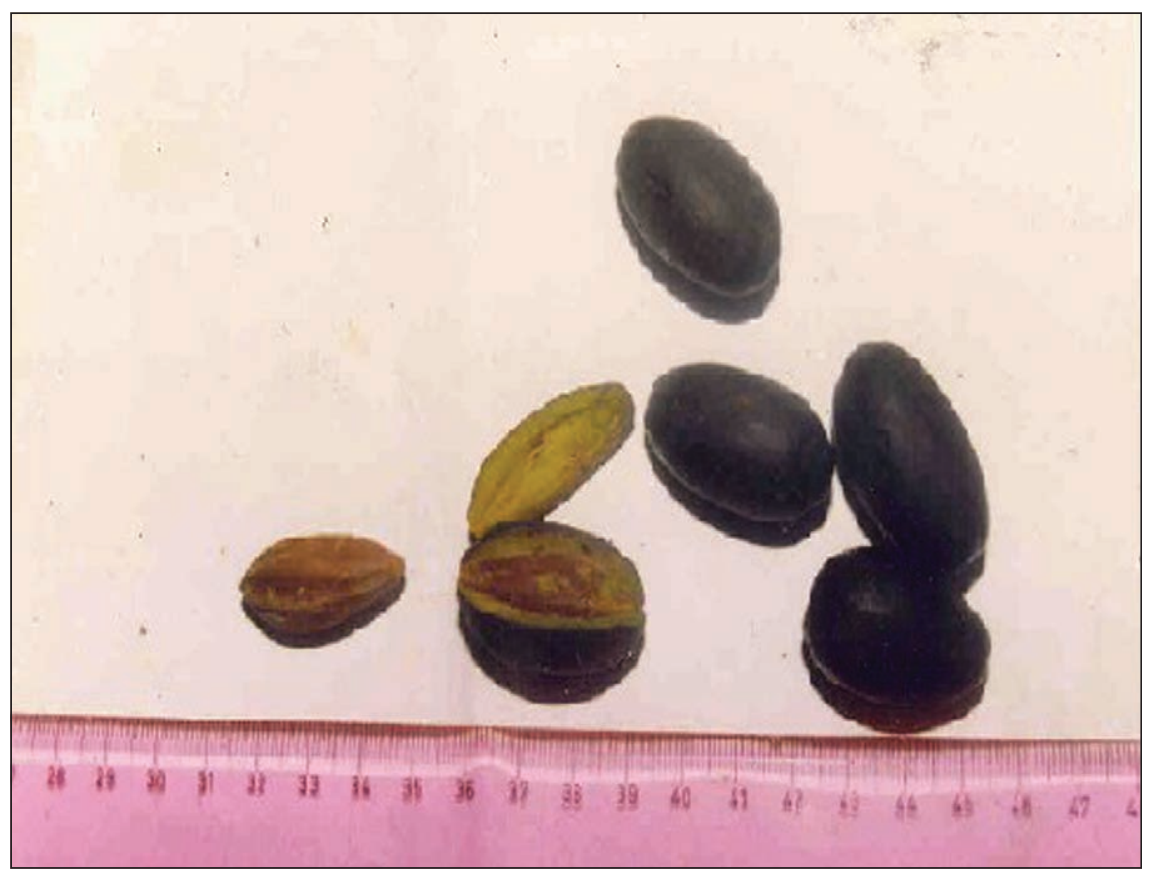

Figure 7. Fruits de Canarium schweinfurthii Engl.

\section{Transformation}

\section{Conservation des fruits ramollis}

La conservation des fruits de l'aiélé en solution est possible et les conserves mises au point (figure 9) restent stables pendant cinq mois. La méthode de ramollissement des fruits à l'intérieur des bocaux à l'aide de la solution de couverture engendre moins de pertes que la méthode pour laquelle le ramollissement des fruits se fait dans un bain d'eau à $45^{\circ} \mathrm{C}$ avant introduction dans les bocaux de conservation. L'essentiel des transferts de matières entre la pulpe des fruits et la solution de couverture s'effectue pendant les traitements thermiques et les quatre jours de mise en conserve des fruits. Les tests sensoriels des fruits montrent que la couleur des pulpes des fruits ramollis et non conservés ainsi que leur texture restent les plus appréciées, tandis que l'odeur et le goût des fruits conservés par la méthode de ramollissement des fruits à l'intérieur des bocaux à l'aide de la solution contenant du chlorure de sodium plaisent aux dégustateurs $[19,20]$.

\section{Valorisation des fruits}

Les fruits de l'aiélé subissent les étapes suivantes pour la production de l'huile: triage, lavage, ramollissement de la pulpe, dépulpage, extraction de I'huile. Même durcie, la pulpe des fruits de l'aiélé peut être utilisée pour la production $\mathrm{d}$ 'huile. La pulpe durcie à $70{ }^{\circ} \mathrm{C}$ a une teneur en huile assez élevée due au traitement à l'eau à chaud qui facilite l'extraction. La qualité de cette huile est proche de celle issue de la pulpe crue et de la pulpe ramollie. Les huiles de la pulpe durcie après stockage des fruits se caractérisent par un taux d'acides gras libres plus élevé comparé à l'huile de pulpe des fruits crus frais [21]. Les tableaux 5 et 6 donnent quelques caractéristiques des huiles des fruits de l'aiélé.

\section{Applications}

Des différentes analyses déjà effectuées sur les différentes huiles végétales, et plusieurs applications potentielles sont possibles. Au Nigeria, dans le cadre de la valorisation des oléagineux non conventionnels, des chercheurs ont investigué sur les possibilités de fabrication de la peinture, du cirage, des laits de beauté et des shampoings à partir de l'huile des fruits de $C$. schweinfurthii. L'accent est également mis sur les utilisations de cette huile comme biocarburant. Les études montrent que I'huile essentielle de C. schweinfurthii pourrait être un agent naturel antimicrobien et antioxydant. II existe également des possibilités d'utilisation en pharmacologie [1, 2].

\section{SAFOU (Dacryodes edulis)}

\section{Production}

Le safoutier appartient à la famille des burséracées. C'est l'un des rares arbres forestiers à 


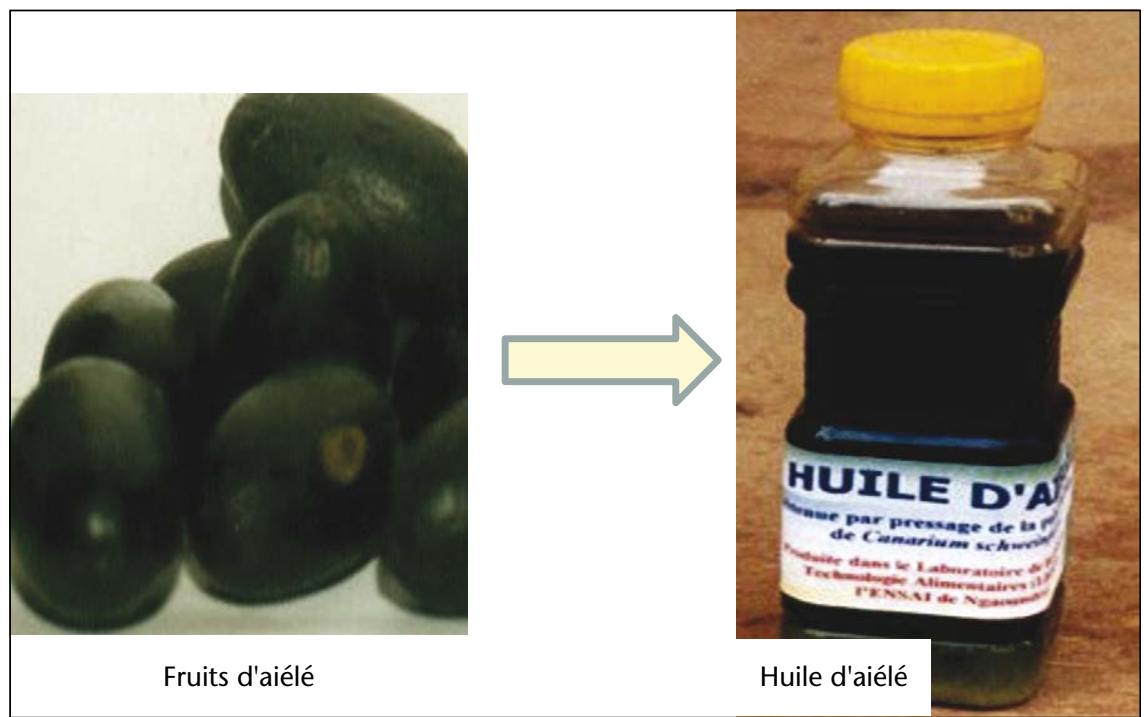

Figure 8. Transformation des fruits d'aielé en huile végétale.

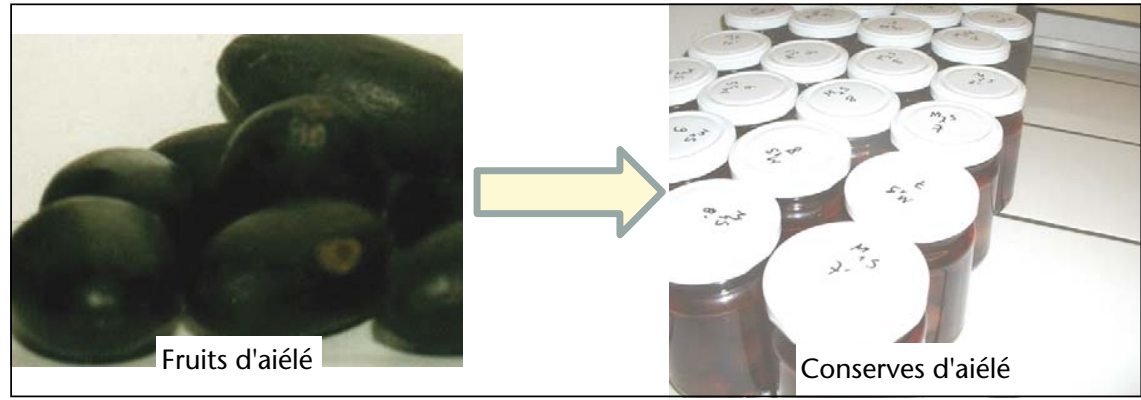

Figure 9. Transformation des fruits d'aielé en conserves.

vertus multiples de la forêt dense tropicale. Malheureusement, sa domestication vient à peine de commencer. La production tardive de la plante vient du fait qu'elle est à peine connue en Afrique (quelques pays de I'Afrique Centrale et Occidentale) et totalement inconnue ailleurs. À première vue, ce retard résiderait dans le manque des données scientifiques sur la qualité et la quantité

de l'huile du fruit. Un safoutier peut produire 7000 à 10500 fruits par arbre. La production est estimée à $223 \mathrm{~kg}$ de fruit par arbre, ce qui conduit à 40-50 kg d'huile par arbre si l'on retient un rendement d'extraction d'huile de $18 \%$ par rapport à la matière frầche. Notons cependant que le safoutier peut conduire à des productions de l'ordre de 4 à 5 tonnes d'huile par hectare.

Tableau 6. Caractéristiques chimiques des huiles des pulpes des fruits de l'aiélé.

\begin{tabular}{|lrccc|}
\hline & Crus & $\begin{array}{l}\text { Ramollis à 45 } \\
\text { pendant } \\
\text { ' C } \\
\text { 40 minutes }\end{array}$ & $\begin{array}{l}\text { Durcis à } \\
\text { I'eau }\end{array}$ & $\begin{array}{l}\text { Durcis après } \\
\text { stockage }\end{array}$ \\
\hline Indices d'acide (mg KOH/g lipide) & $10,2 \pm 0,2$ & $10,3 \pm 0,4$ & $10,8 \pm 1,0$ & $12,1 \pm 0,1$ \\
\hline Indice de peroxyde & $7,8 \pm 0,1$ & $10,1 \pm 0,1$ & $11,1 \pm 0,2$ & $12,3 \pm 0,3$ \\
\hline Indices d'iode (g d'iode/g lipide) & $86,5 \pm 1,1$ & $71,4 \pm 1,1$ & $68,4 \pm 1,0$ & $80,8 \pm 1,3$ \\
\hline Indices de saponification & $184,8 \pm 0,1$ & $188,3 \pm 0,3$ & $202,3 \pm 0,1$ & $182,7 \pm 0,2$ \\
\hline Insaponifiables (g/100 g) & $1,1 \pm 0,1$ & $1,2 \pm 0,1$ & $1,3 \pm 0,1$ & $1,0 \pm 0,1$ \\
\hline
\end{tabular}

\section{Teneur en huile}

La teneur en huile varie suivant la source $d$ 'huile. L'avocat fournit la teneur d'huile la plus élévée alors que le maïs fournie la teneur la plus faible. Ceci est dû au fait que c'est le germe de maïs qui contient I'huile. Les huiles non conventionnelles comme le safou et les corps gras, les courges et le djansang ont des teneurs en huile supérieures à $50 \%$. Ceci suggère la possibilité de mettre en place une petite huilerie à base de ces plantes. 


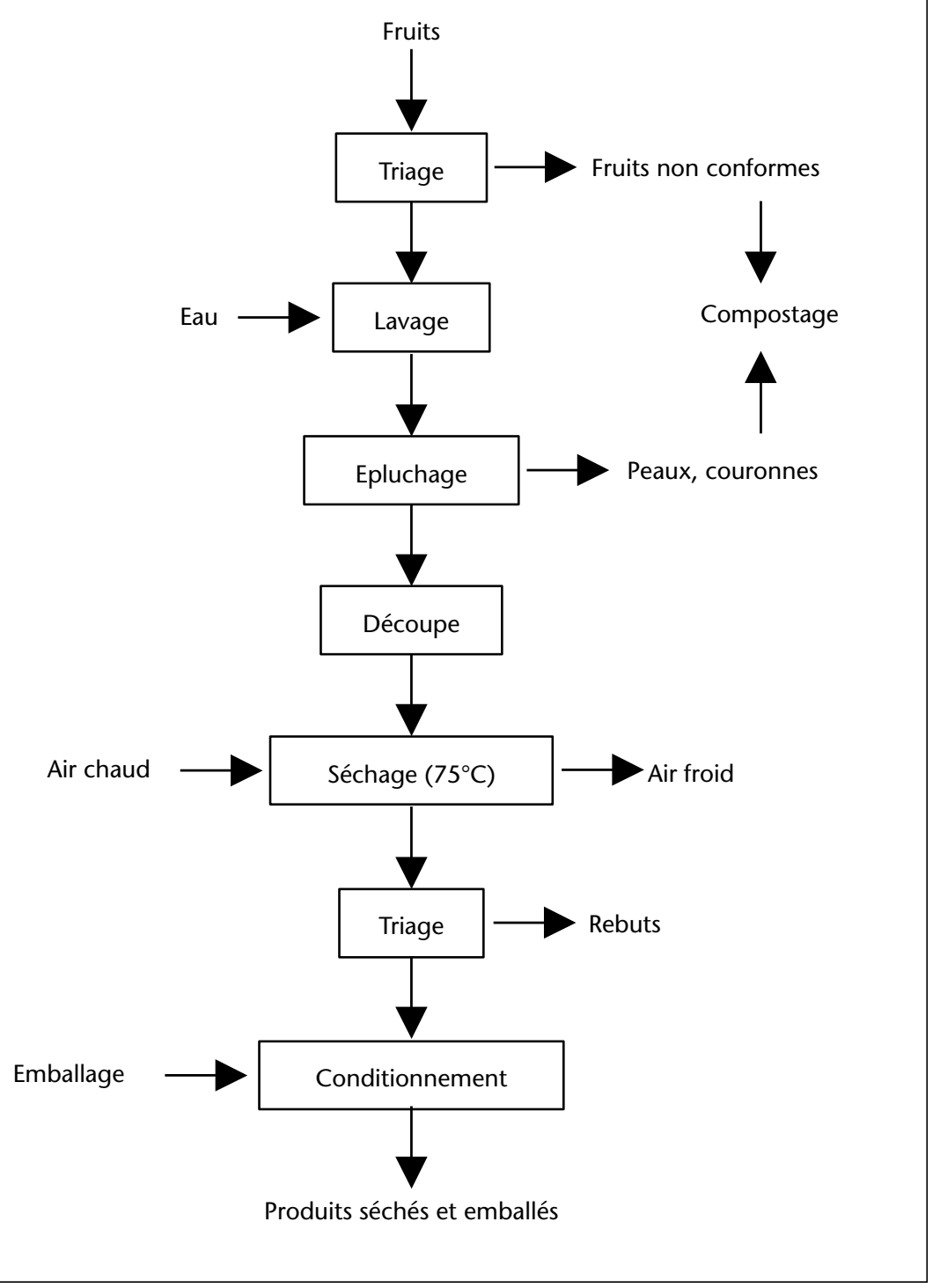

Figure 10. Procédé de transformation du safou en vue d'obtenir un produit séché.

\section{Acides gras totaux}

Les acides gras totaux varient en fonction de la source d'huile. Le soja présente la valeur la plus élevée alors que la courge blanche présente la valeur la plus faible. Cette variation est due à la sélection variétale et aux conditions d'extraction. Parmi, les huiles non conventionnelles, le Canarium et la courge rouge présentent les valeurs élevées comparées aux autres oléagineux africains étudiés.

\section{Composition en acides gras}

Les oléagineux étudiés présentent différents acides gras au nombre de 15 (tableau 7). Leur taux varie en fonction du type d'huile végétale. siccatives. Les huiles tri-insaturées (djansang) contiennent plus de $50 \%$ d'acide linolénique. Les acides laurique, palmitique, oléique, et stéarique sont les acides gras principaux communément trouvés dans les oléagineux africains. Les acides gras secondaires sont les acides myristique et linolénique.

\section{Composition en triglycérides}

Le tableau 8 donne les valeurs de chacun des triglycérides séparés. Les 15 oléagineux africains possèdent des triglycérides en fonction de leurs types d'huiles.

Les huiles de type laurique possèdent quatre principaux triglycérides (LaLaLa, LaLaM, CiLaLa, CyLaLa), et 3 triglycérides secondaires (LaMM, CyPS, CyCiLa).

Les huiles de type palmitique, stéarique, oléique et linoléique possèdent cinq triglycérides principaux : OOO, POO, LOO, LLL et PLO et trois triglycérides secondaires: LOL, POP, soO.

Les huiles de type tri-insaturées possèdent deux triglycérides principaux (EEL, EEE) et 6 triglycérides secondaires. Le djansang a le taux EEE le plus élevé (15,9\%).

\section{Conclusion}

Les grandes cultures oléaprotéagineuses telles que le palmier, le coton et l'arachide sont en constante évolution sur le triple plan de la production, de la transformation et des utilisations. Le karité se positionne comme une culture émergente avec son incorporation dans le chocolat et les usages cosmétiques. Les cultures domestiques comme le Canarium et le safou sont en train de créer un renouveau de la culture oléagineuse africaine. Ces fruits oléagineux sont valorisés sous forme de conserves (Canarium) et fruits séchés (safou). Les compositions en acides gras et en triglycérides ont permis de caractériser les huiles végétales africaines.

La production des huiles des oléagineux en Afrique reste dans la majorité des cas encore à l'échelle traditionnelle et constitue une source de revenus pour les populations. La qualité des huiles n'est pas uniforme. Les huiles produites sont utilisées dans I'alimentation, la pharmacopée et le cosmétique.

La conservation du Canarium a atteint un niveau tel qu'il est possible de mettre en place une petite unité de conserverie. Le problème sera celui de la mise en place des circuits de collecte et de caractérisation de la matière première afin de connaître les origines d'appellations contrôlées. 


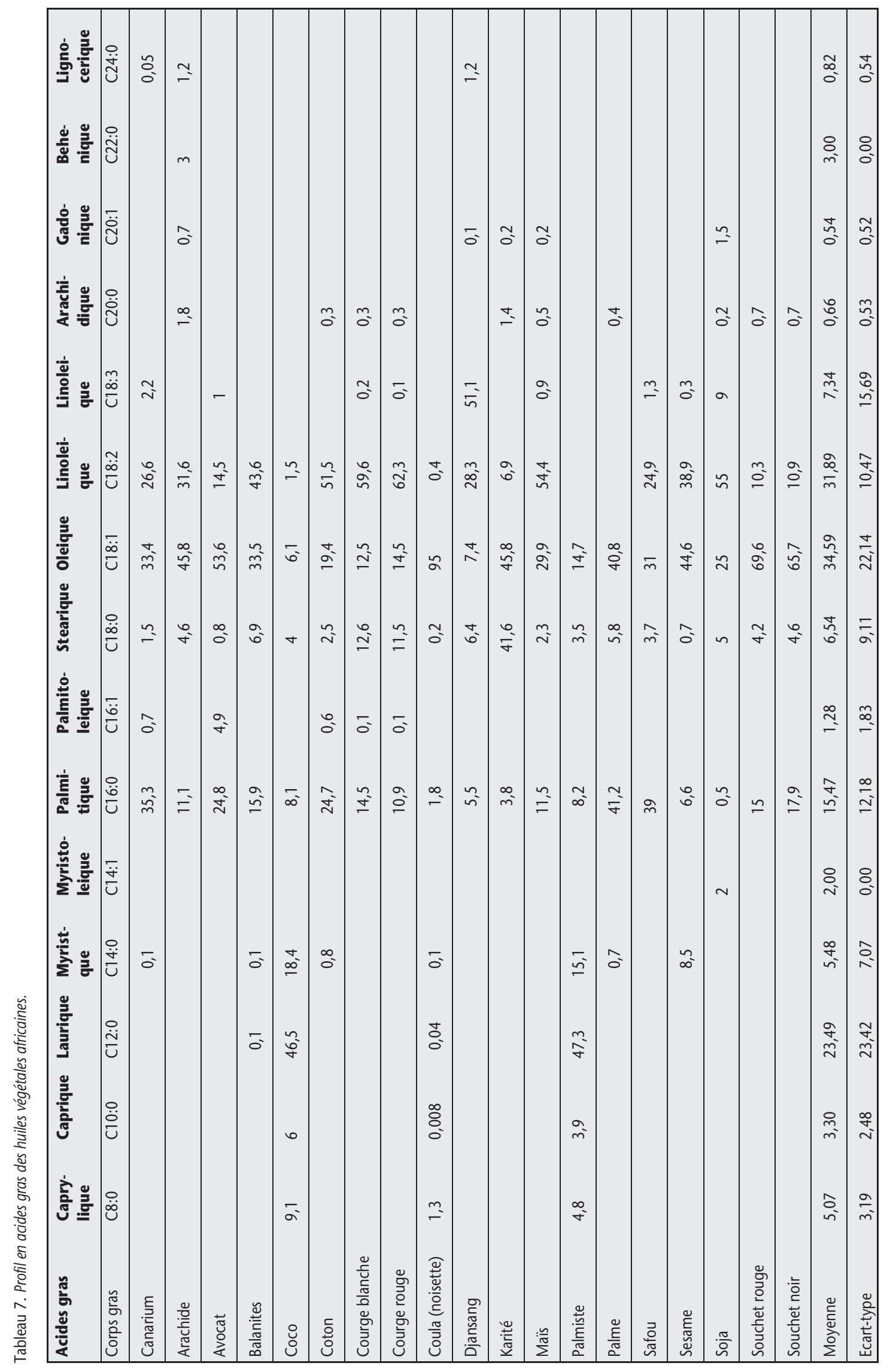




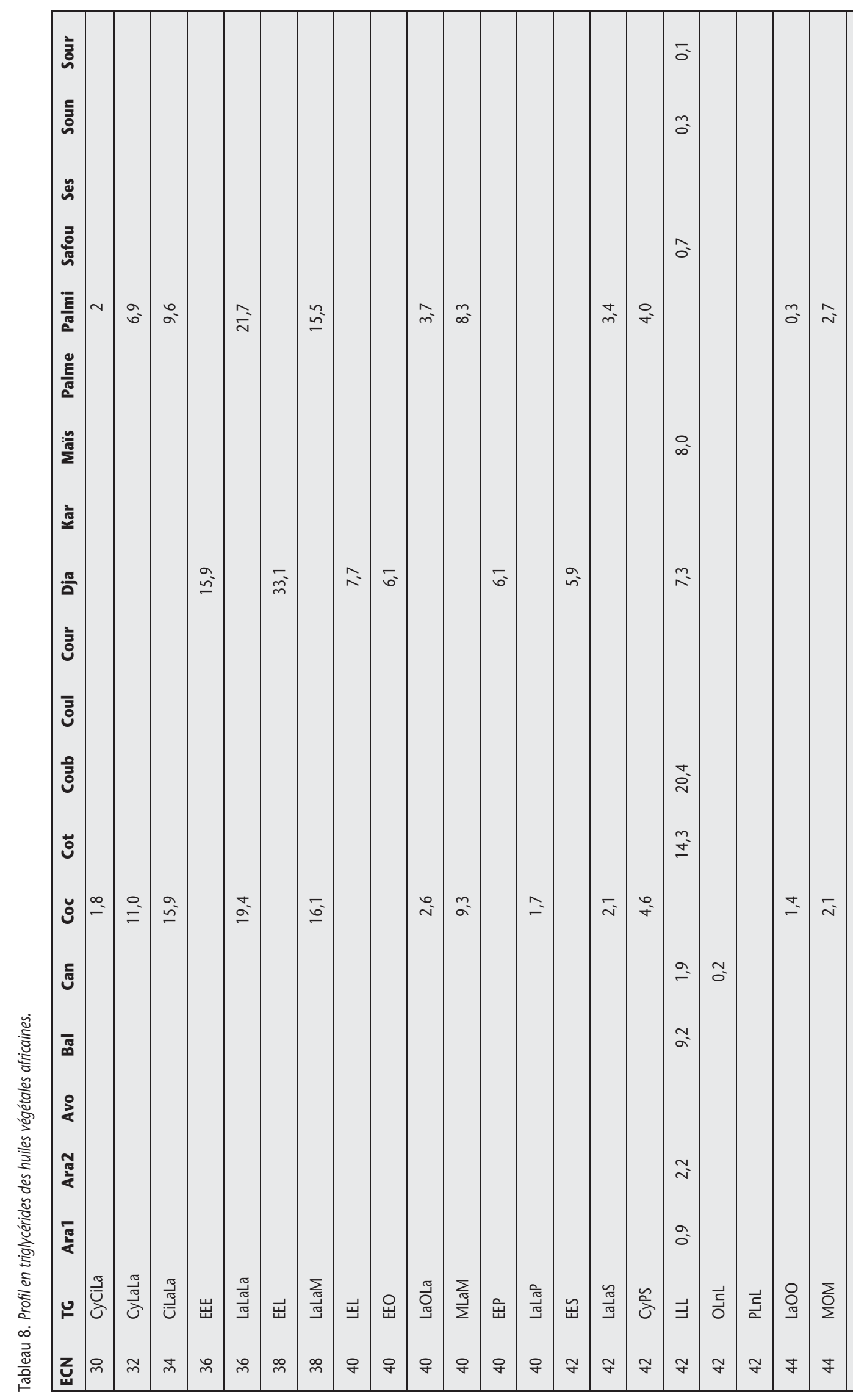




\begin{tabular}{|c|c|c|c|c|c|c|c|c|c|c|c|c|c|c|c|c|c|c|c|c|c|c|c|c|}
\hline ڤे & & & $\hat{i}$ & & $\stackrel{0}{-}$ & & & & $\stackrel{\Xi}{\circ}$ & & $\stackrel{n}{\sim}$ & $\cong$ & & & $\stackrel{n}{n}$ & & & $\stackrel{q}{\sigma}$ & $\bar{o}$ & $\approx$ & $\stackrel{\sim}{\sim}$ & $\stackrel{\forall}{0}$ & & $m$ \\
\hline 童 & & & $\approx$ & & $\stackrel{0}{=}$ & & & & 茫 & & $\tilde{n}$ & $\stackrel{m}{=}$ & & & $\bar{\sim}$ & & & in & & $\approx$ & $\tilde{m}$ & $\hat{o}$ & & $\hat{v}$ \\
\hline 衣 & & & $\stackrel{0}{=}$ & & $\bar{\sigma}$ & & & & $\grave{v}$ & & $\begin{array}{l}\stackrel{n}{\infty} \\
\sim^{0}\end{array}$ & $\tilde{o}^{-}$ & & & वे & & 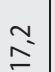 & $\tilde{\sigma}$ & $\hat{o}$ & $\cong$ & $\stackrel{\infty}{=}$ & $\tilde{0}$ & & \\
\hline$\frac{\overline{\underline{\varepsilon}}}{\bar{E}}$ & $\bar{m}$ & $\underset{\Xi}{\Xi}$ & 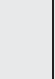 & & & & $\cong$ & $\stackrel{g}{\Rightarrow}$ & & & & & & & & & $\hat{o}$ & & & & & & & \\
\hline 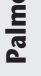 & & & $\begin{array}{l}0 \\
0\end{array}$ & & $\hat{\mathrm{i}}$ & & & & $\stackrel{\infty}{\sim}$ & & $\stackrel{m}{\circ}$ & $\hat{\sigma}$ & - & & $\stackrel{0}{m}$ & & $\hat{\bar{i}}$ & $\stackrel{m}{m}_{m}^{m}$ & $\hat{\sim}$ & $\stackrel{m}{m}$ & $\tilde{0}$ & $\hat{\sigma}$ & $\stackrel{0}{=}$ & \\
\hline 滔 & & & $\frac{n}{\sim}$ & & $\begin{array}{l}\bullet \\
\infty\end{array}$ & & & & 이 & & $\begin{array}{l}\infty \\
\stackrel{\infty}{ \pm}\end{array}$ & $\bar{v}$ & & & $\widehat{\infty}$ & & $\underset{\nabla}{\sigma}$ & $\hat{0}$ & & & $\bar{v}$ & & & \begin{tabular}{l}
0 \\
\hdashline
\end{tabular} \\
\hline " & & & $\overline{0}$ & & & & & & & & & & & $\stackrel{\infty}{=}$ & & $\stackrel{ \pm}{ \pm}$ & & & & & & & & \\
\hline 言 & & & 으 & & $\begin{array}{l}0 \\
\tilde{\sim}\end{array}$ & & & & $\stackrel{0}{n}$ & & $\stackrel{0}{i}$ & $\stackrel{\sim}{\varkappa_{\infty}^{-}}$ & & & $\stackrel{\Xi}{0}$ & & & & & $\stackrel{0}{\circ}$ & $\nabla$ & & & $\tilde{0}$ \\
\hline ڤै & & & $\cong$ & & $\begin{array}{l}\stackrel{a}{\mathbf{N}} \\
\text { a }\end{array}$ & & & & $\stackrel{\infty}{\stackrel{\infty}{\forall}}$ & & $\begin{array}{l}m \\
\mathscr{c}^{-}\end{array}$ & 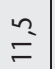 & $\hat{0}$ & $\approx$ & $\stackrel{m}{\sim}$ & & तै & $\stackrel{\sim}{\circ}$ & $\tilde{o}$ & $\Xi$ & $=$ & & & \\
\hline ๖ & $\stackrel{m}{=}$ & $\stackrel{\circ}{=}$ & & & & & $\stackrel{\circ}{i}$ & $\hat{0}$ & & & & & & & & & $\tilde{m}$ & & & & & & & \\
\hline స్ & & & $\tilde{m}$ & & & & & & & & 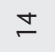 & $\stackrel{\sim}{\sim}$ & & & $\stackrel{\sim}{\sim}$ & & $\hat{a}$ & $\stackrel{\nabla}{\sim}$ & $\begin{array}{l}\mathscr{\sigma} \\
\underset{\sigma}{*}\end{array}$ & $\tilde{c}_{0}^{\prime}$ & $\stackrel{m}{=}$ & & & \\
\hline$\overline{్ ల ్ ~}$ & & & $\begin{array}{l}\infty \\
\stackrel{n}{n}\end{array}$ & & 으 & & & & 으 & & $\stackrel{\infty^{-}}{\leftarrow}$ & $m$ & & & $\stackrel{n}{\sim}$ & & & $\hat{n}$ & & Ғ & $\stackrel{و}{\forall}$ & & & $\stackrel{m}{=}$ \\
\hline$\stackrel{8}{\gtrless}$ & & & $\underset{\sim}{\sim}$ & & & & & & $\Xi$ & & $a^{m}$ & $\stackrel{0}{=}$ & & & $\begin{array}{l}\simeq \\
\sim^{0} \\
\overbrace{}^{2}\end{array}$ & $\tilde{\sigma}$ & $\frac{m}{\bar{v}}$ & $\underset{\infty}{\forall}$ & $\Xi$ & $\tilde{o}$ & & & & \\
\hline ฮู้ & & & $\hat{\tilde{n}}$ & & $\stackrel{m}{\sim}$ & & & & $\stackrel{\stackrel{\sim}{\sim}}{\bar{N}}$ & & $\underset{\simeq}{\simeq}$ & $\dddot{r}$ & & & $\stackrel{a}{\check{n}}$ & & $\stackrel{\nabla}{\circ}$ & $\stackrel{\Xi}{ \pm}$ & & $\bar{m}$ & $\stackrel{i}{i}$ & & & $\stackrel{\forall}{\circ}$ \\
\hline হ & & & $\stackrel{\infty}{N}$ & & $\stackrel{m}{\sim}$ & & & & $\stackrel{\circ}{\circ}$ & & 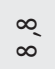 & ò & & & $\begin{array}{l}\infty \\
\vdots \\
\mathbf{N}^{-1}\end{array}$ & & $\begin{array}{l}\stackrel{0}{\tilde{m}} \\
\tilde{T}^{-}\end{array}$ & $\stackrel{0}{\circ}$ & & $\stackrel{\infty}{n^{n}}$ & $=$ & & & $\stackrel{0}{0}$ \\
\hline ي & $\sum_{a}$ & $\stackrel{\widetilde{\Xi}}{\vec{\Sigma}}$ & $\overrightarrow{\mathrm{O}}$ & $\begin{array}{l}\text { 오 } \\
\text { ○ }\end{array}$ & $\vec{a}$ & $\underset{ড}{\stackrel{\complement}{c}}$ & о̄ & స్త్ & $\stackrel{\circ}{\circ}$ & о̄ & ธิ & $\stackrel{\grave{a}}{a}$ & оे & $\vec{\Xi}$ & ৪ & $\stackrel{\circ}{\sim}$ & ঃ & оे & à & $\stackrel{\circ}{\circ}$ & $\widetilde{\circ}$ & $\check{a}$ & $\approx \check{\check{a}}$ & 乞̊ \\
\hline z & $\underset{\forall}{ }$ & $\underset{\forall}{ }$ & $\forall$ & J & F & $\forall$ & 아 & $\stackrel{+}{\circ}$ & \% & $\stackrel{\sigma}{*}$ & $\stackrel{+}{+}$ & $\stackrel{+}{+}$ & $\stackrel{+}{*}$ & $\stackrel{+}{\circ}$ & $\stackrel{\infty}{\sim}$ & $\stackrel{\infty}{\sigma}$ & $\stackrel{\infty}{\sim}$ & $\stackrel{\infty}{\sigma}$ & $\stackrel{\infty}{\sim}$ & in & in & in & in & $\approx$ \\
\hline
\end{tabular}




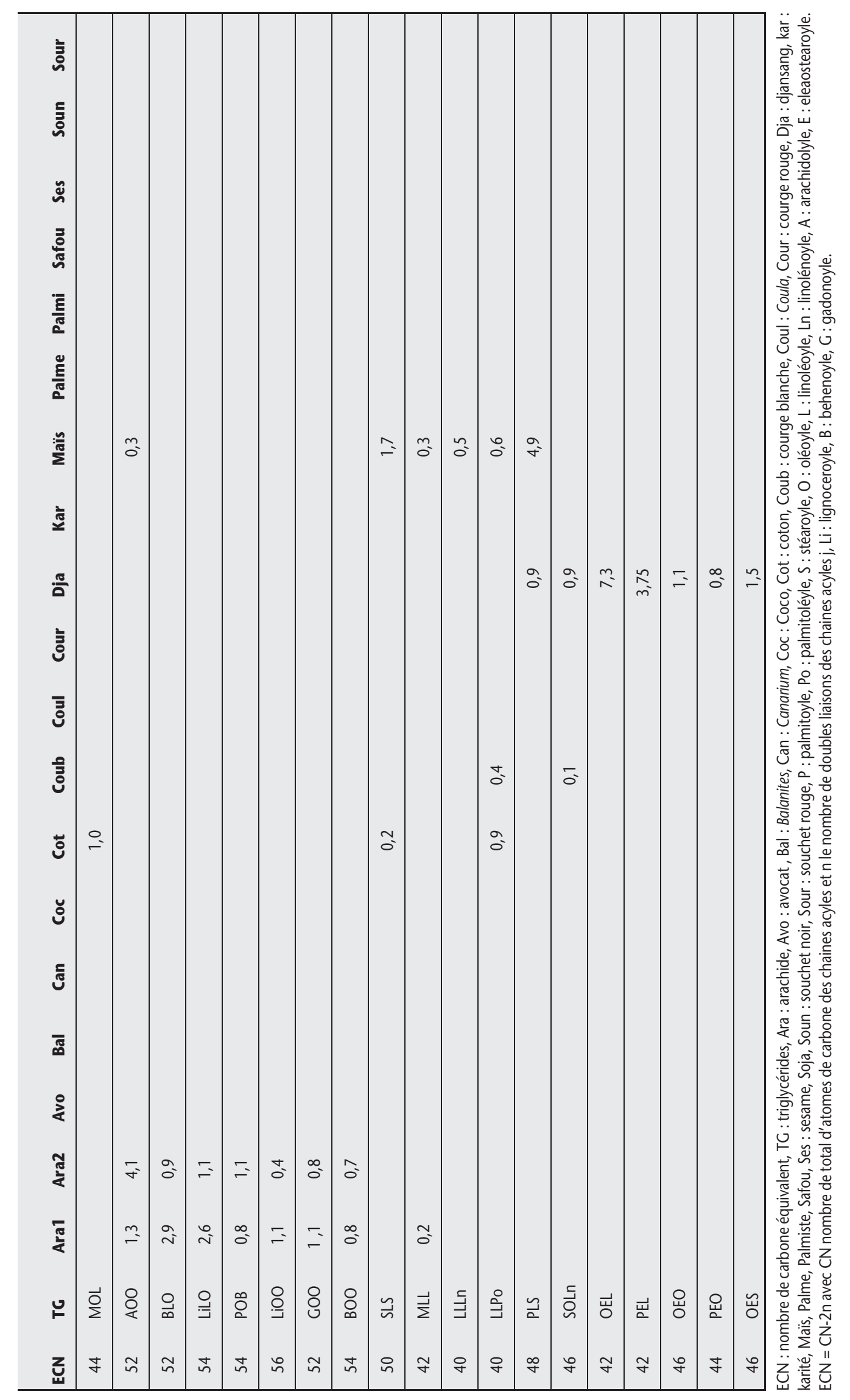


Tableau 8. Légendes des abréviations du tableau 8.

\begin{tabular}{|lll|}
\hline Nom usuel & Nom scientifique & Abréviation \\
\hline Arachide cultivar 1 (Nord Cameroun) & Arachis hypogaea & Ara1 \\
\hline Arachide cultivar 2 (Ouest Cameroun ) & Arachis hypogaea & Ara2 \\
\hline Avocat & Persea americana & Avo \\
\hline Balanite & Balanites aegyptiaca (L.) Del. & Bal \\
\hline Fruit noir & Canarium schweinfurthii Engl. & Can \\
\hline Coco & Cocos nucifera & Coc \\
\hline Coton & Cossypium sp & Cot \\
\hline Courge blanche & Cucurbita Cucumeropsis manii Naudin & Coub \\
\hline Courge rouge & Cucumeropsis manii Naudin & Cour \\
\hline Noisette & Coula edulis & Coul \\
\hline Djansang & Ricinodendron heudelotii & Dja \\
\hline Karité & Vitellaria paradoxa & Kar \\
\hline Maïs & Zea mays & Maïs \\
\hline Palme & Elaeis guineensis Jacq. & Ses \\
\hline Palmiste & Elaeis guineensis & Soun \\
\hline Safou & Dacryodes edulis & Sour \\
\hline Sésame & Sesamum indicum & \\
\hline Soja & Clycine max (L.) & Cyperus esculentus \\
\hline Souchet noir & Cyperus esculentus & \\
\hline Souchet rouge & & \\
\hline
\end{tabular}

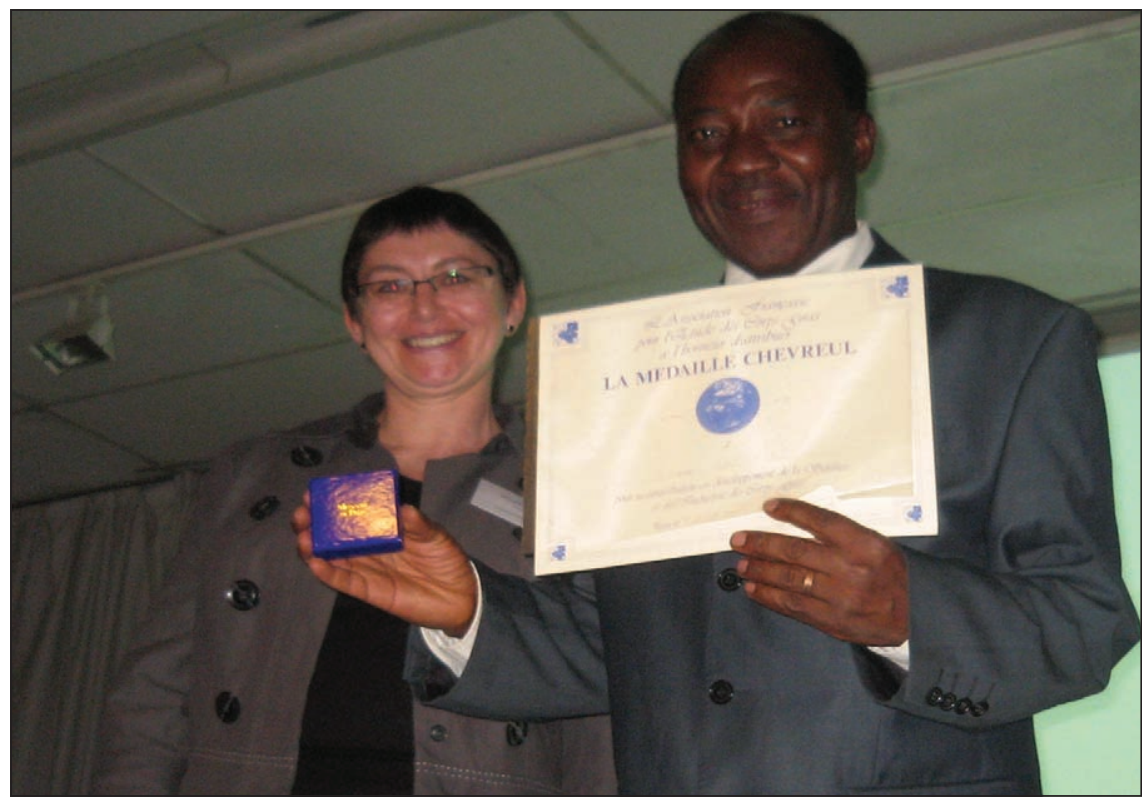

Figure 12. César Kapseu, récipiendaire de la Médaille Chevreul 2009 et et Anne Le Guillou, Présidente de I'AFECG.

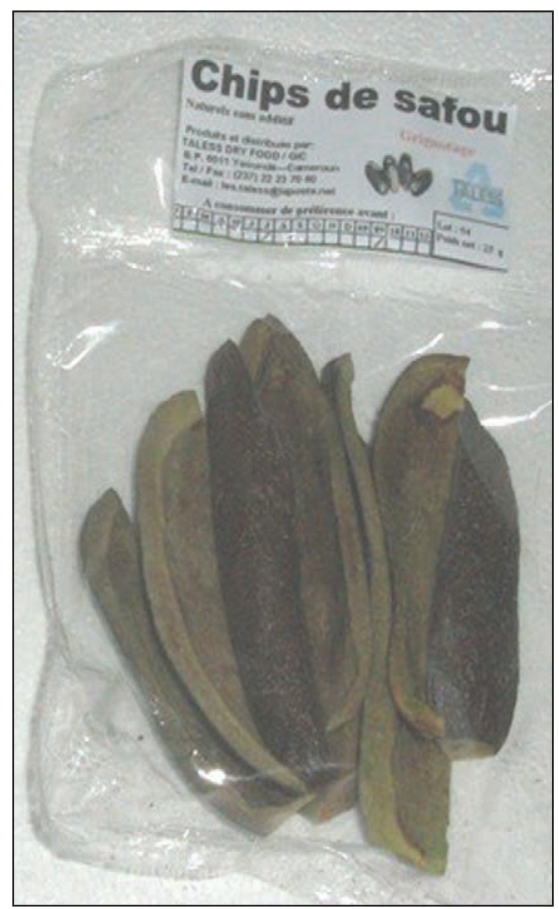

Figure 11. Safou séché (chips).

En ce qui concerne le safou, la mise en place d'une unité de production de produits séchés afin de résoudre le problème de perte postrécolte très élevé dans cette filière ouvre des perspectives intéressantes avec l'appropriation de la technologie par une petite et moyenne entreprise.

Remerciements. Le conférencier remercie Womeni Hilaire, Bup Divine, Tchankou Carine, Nouadjep Serges, Petsoko Mathurin, Atoukam Liliane et Kapchié Virginie pour leur assistance technique. Les remerciements vont également aux organismes qui ont soutenu les différents travaux qui sont résumés dans cet article (Université de Ngaoundéré (Cameroun), AUF (Canada), SCAC (Cameroun), FIS (Suède), AIRE - Développementet IRD (France), ATLAS/AAI (USA)). J'exprime ma gratitude à l'ensemble des collaborateurs de l'Equipe de recherche RESH (Réaction, Extraction, Sucrerie Séchage, Huilerie) pour leur précieuse contribution. La figure 12 présente le récipiendaire de la médaille Chevreul 2009 et la Présidente de l'AFECG.

\section{RÉFÉRENCES}

1. Kengue J, Kapseu C, Kayem G]. Actes du $3^{e}$ Séminaire International sur la valorisation $d u$ Safoutier et autre oléagineux non conventionnel. Yaoundé: Presses Universitaires d'Afrique, 2002 ; 426 pages. 
2. Kapseu C, Kayem J. Actes du séminaire International sur le séchage et sur la valorisation du karité et de l'aiélé. Yaoundé : Presses Universitaires, $2000 ; 410$ pages

3. Kapseu C. Improvement of new sources of vegetable oil in Cameroon. Final technical report of ATLAS/AAI project, Dairy Science Department, OARDC/OSU, Wooster, Ohio, USA, 1993, $60 \mathrm{p}$.

4. Kapseu C, Kayem J. Actes du $2^{\text {ème }}$ séminaire International sur la valorisation du safoutier, Ngaoundéré. Presses Universitaires de Yaoundé, 1998, 444 p.

5. Womeni HM, Ndjouenkeu R, Kapseu C, Parmentier $\mathrm{M}$, Fanni J. Application du procédé séchage-friture aux amandes de karité: influence sur les indices chimiques de qualité et les propriétés de fusion du beurre. OCL $2006 ; 13: 1-5$.

6. Womeni HM, Ndjouenkeu R, Kapseu C, Tchouanguep Mbiapo F, Parmentier M, Fanni J. Effet de la Cuisson et du Séchage des Noix de Karité (Butyrospermum parkii (G. Don) Kostchy sur la Qualité du Beurre. Tropicultura 2006 ; 24 : 175-82.

7. Kapseu C, Nketcha PR, Bornaz S, Parmentier M, Dirand M. Influence des conditions locales de production et de stockage sur les propriétés de I'huile de palme du Cameroun et ses fractions. Gennaio 2004 ; LXXV : 29-34.

8. Kapseu C. Physico-Chimie de la formation de dépôts solides à partir de l'huile de coton. Doctorat d'Etat ès Sciences, ENSIC-INPL, Nancy, France, Novembre 1989, 216 p.

9. Ndjeunga J, Ntare BR, Schilling R. Perspectives globales et régionales du marché de l'arachide : compétitivité des producteurs africains. In: Ntare Bonny R, Mayeux Alain, Waliyar Farid (Eds). Conservation, évaluation et diffusion du matériel arachide et production et diffusion de semences de base en Afrique de l'ouest : comptes rendus de I'atelier de restitution du projet Germplasm arachide, 22-24 avril 2002, Bamako, Mali. Patancheru : ICRISAT, p. 55-75. Atelier de restitution du projet Germplasm arachide, 2002-04-22/2002-04-25, Bamako, Mali, 2003.

10. Schilling R, Dimanche P. L'arachide dans le monde et en Afrique : quelques données économiques récentes. OCL $1994 ; 1$ : 8-11.

11. Womeni HM, Ndjouenkeu R, Kapseu C, Tchouanguep Mbiapo F, Parmentier M, Fanni J. Séchage des Amandes de Karité et Qualité du Beurre: Impact du Séchage Traditionnel au Soleil. Tropicultura 2007 ; 25 : 240-7.

12. Womeni HM, Ndjouenkeu R, Kapseu C, et al. Application du procédé séchage-friture aux amandes de karité : influence sur les indices chimiques de qualité et les propriétés de fusion du beurre. OCL 2006 ; 24 : 175-82.
13. Bup Nde D, Kapseu C, Tenin D, Kuitche A, Fon Abi C, Tchiégang C. Variation of the Physical Properties of Sheanut (Vitellaria paradoxa Gaertn.) Kernels during Convective Drying. Int J Food Engineering 2008 ; 4 : 1-19.

14. Mohagir C, Kapseu R, Kamga et al. Optimization of some pre-treatments involved in the press extraction of Shea (Vitellaria paradoxa Gaertner F.) butter. Asian Journal of Applied Sciences 2009.

15. Njoukam R. L'Arbre aux Fruits Noirs : I'aiélé. Flamboyant $1998 ; 46: 11-5$.

16. Tchouamo IR, Tchoumboué J, Punta JY, Njoukam R. L'aiélé (Canarium schweinfurthii Engl.) : plante à usages multiples en Afrique. La Rivista Italiana Delle Sostanze Grassa 2000 ; LXXVII : 77-81.

17. Vivien J, Faure JJ. Arbres des forêts denses d'Afrique Centrale. République du Cameroun ; Ministère français des relations extérieures, de la coopération et du développement, France ; 1986.

18. Tchiegang C, Kapchie Noutchougoue V, Kapseu C, Parmentier M. Influence du temps, de la température et des conditions de stockage sur le ramollissement des fruits de l'aiélé (Canarium schweinfurthii Engl.). J Food Eng $2001 ; 42: 63-8$.

19. Tchiegang L. Tchatchouang Ngasop C. Kapseu, Parmentier M. Etude comparative du ramollissement des fruits de Canarium schweinfurthii Engl. par voie humide et voie sèche. J Food Eng $2004 ; 62: 69-77$.

20. Tchiegang C, Kapseu C, Tchatchouang Ngasop $L$, Parmentier M. Etude de quelques paramètres physico-chimiques des fruits ramollis de Canarium schweinfurthii Engl. Pendant la conservation dans différentes solutions acides. Sciences des Aliments $2002 ; 22$ : 289-303.

21. Tchiegang C, Kamga R, Kapseu C. African black olive (Canarium schweinfurthii Engl.) : a novel ingredient for small scales traditional oil production. La Rivista Italiana Delle Sostanze Grasse $1998 ; 75$ : 565-7.

22. Tchankou Leudeu BC. Etude sur des jeunes rats mâles des propriétés nutritionnelles des huiles de Canarium schweinfurthii Engl., Dacryodes edulis (G. Don) H. J. Lam, Ricinodendron heudelotii (Bail.) Pierre ex Pax, et Tetracarpidium conophorum Müll. Arg. Hutch. and Dalz. Thèse de Doctorat, Ecole Nationale Supérieure des Sciences Agro-Industrielles/Université de Ngaoundéré (Cameroun); 2006; 259 p.

23. Tchankou Leudeu BV, Tchiegang C, Dzondo Gadet M, et al. Effect of Canarium schweinfurthii and Dacryodes edulis oils on Blood Lipids, Lipid Peroxidation and Oxidative Stress in Rats. Journal of Food Technology $2006 ; 4$ : 275-82.
24. Tchankou Leudeu BC, Tchiégang C, Barbé F, Nicolas B, Guéant JL. Ricinodendron heutelotii (Bail.) or Tetracarpidium conophorum Müll. oils fed to male rats lower blood lipids. Nutrition Research 2009 ; 29 : 503-9.

25. Tchiégang C, Kapchié NV, Mbofung CM, Kapseu C. Variations dans les caractéristiques physico-chimiques des fruits de l'aiélé (Canarium schweinfurthii Engl.) de différentes provenances du Cameroun. La Rivista Italiana Delle Sostanze Grasse 2000 ; 78 : 537-42.

26. Kapseu C, Parmentier M, Kayem G], Schufferner Dirand M. Fatty acid and triglycerides of Canarium schweinfurthii Engl. Fruits. International Tree Crops Journal $1996 ; 19$ : 77-86.

27. Noumi GB, Ngameni E, Kapseu C, Parmentier M.Variation de la composition en acides gras et en triglycérides de l'huile des fruits de l'aiélé en fonction des conditions d'extraction et de la couleur du fruit. Rivista Italiana Delle Sostanze Grasse LXXIX : 315-321

28. Kapseu C, Jiokap Nono Y, Parmentier M, Dirand $\mathrm{M}$, Dellacherie J. Acides gras et triglycerides du beurre de karite du Cameroun. La Rivista italiana delle sostanze grasse 2001 ; LXXVIII : 31-4.

29. Kapseu C, Mapongmetsem PM, Silou T, Roques M. Physico-chimie des fruits du safoutier (Dacryodes edulis) camerounais. Tropicultura 1998-99; 16-17 : 37-42.

30. Kapseu C. Quelques propriétés physico chimiques des huiles des oléagineux nonconventionnelles. La Rivista Italiana Delle Sostanze Grasse (Italie) 1998 ; LXXV : 295-8.

31. Kapseu C, Parmentier M. Composition en acides gras de quelques huiles végétales du Cameroun. Sciences des Aliments $1997 ; 17$ : 85-92.

32. Kapseu C, Mbofung CMF, Kayem G). Acides gras et triglycérides des huiles de fruits de Cyperus esculentus et Balanites aegyptiaca. Sciences des Aliments 1997 ; 17: 531-7.

33. Kapseu C, Tchiegang C, Dellacherie J, Dirand M. Huiles des noix de coco et de palmiste du Cameroun : sources d'acide gras laurique. Sostanze Grasse 1997 ; 74 : 205-7.

34. Kapseu C, Houriez J, Roques M. Acides gras et triglycérides de I'huile de mais du Cameroun (Zea mays Lin.). Sostanze Grasse 1997 ; LXXIV : 461-3.

35. Tchiegang C, Kapseu C. Influence de l'humidité, des teneurs en coque et en gossypol total sur la couleur de la farine des amandes des graines de cotonnier. J Food Eng 1997; 34 : 259-70.

36. Kapseu C, Tchiegang C. Composition de l'huile des fruits de deux cultivars de safou au Cameroun. Fruits $1996 ; 51: 185-91$. 
37. Kapseu C, Kamga R, Tchatchueng JB, Kayem G). Triacylglycerols and fatty acids of the major tropical oils. Transactions of Zimbabwe Science. Transformation of the Zimbabwe Scientific Association, nº 70, $1996: 28-32$.

38. Kapseu C, Tchiegang C. Chemical Properties of Ricinodendron heudelotii (Bail.) seed oil. Journal of Food Lipids $1995 ; 2$ : 87-98.

39. Kapseu C, Kayem G), Balesdent D. Compositions d'huiles de palme et ses fractions. Ann Fac Sci, H.S.n ${ }^{\circ}$ 3. Chim Et Sci Nat $1994: 12-25$.
40. Kapseu C, Kamga R, Tchatchueng JB. Triacylglycerols and fatty acids composition of egusi seed oil (Cucumeropsis Mannii Naudin). Grasas y Aceites 1993 ; 65 : 354-6.

41. Kapseu C, Kamga R, Tchatchueng JB, Kayem G). Etude Comparative de la composition des huiles de maïs et d'arachide. Revue Française des Corps Gras $1992 ; 7 / 8: 215-8$.

42. Kapseu C, Kayem G), Balesdent D, Dirand M. Contrôle du raffinage de l'huile de coton par HPLC. Sciences et techniques $1992 ; 2$ : 37-41 (RAIST).
43. Balesdent D, Kapseu C, Kayem G). Caractérisation des triglycérides de I'huile de coton par chromatographie liquide haute performance (C.L.H.P) en une seule étape. Revue Française des Corps Gras $1989 ; 1: 27-33$.

44. Tchiégang C, Tchankou BC, Kapseu C, Fomethe $A$, Parmentier $M$. Influence of water content of the pulp extraction and the physiochemical properties of canarium schweinfurtii oil. Eur / Lipid Sci Technol $2004 ; 106: 613-20$. 